\title{
Sustainability and Sustainable Development: Chance of System Failure Due to Event Characteristics
}

\author{
Charles Arvid Backman \\ Department of Business and Office Administration, Grande Prairie College, Grande Prairie, Canada \\ Email address: \\ cbackman $@$ gprc.ab.ca, cabackman $@$ eastlink.ca, cbackman $@$ telus.net

\section{To cite this article:} \\ Charles Arvid Backman. Sustainability and Sustainable Development: Chance of System Failure Due to Event Characteristics. Journal of \\ Energy and Natural Resources. Vol. 8, No. 1, 2019, pp. 18-29. doi: 10.11648/j.jenr.20190801.14
}

Received: January 20, 2019; Accepted: March 15, 2019; Published: April 28, 2019

\begin{abstract}
While performance along social, environment and economic dimensions has gained traction in business and society, the focus has been on system continuity. Because of the different interpretations which can be attached to sustainability or sustainable development, consensus on appropriate goals of the system, whatever the system might be, may be more difficult to develop than consensus on what is not wanted - such as a system failure. System failure in this context is meant to convey a system's inability to adapt to changes in the external or internal environments brought on by an event that disturbs the equilibrium of forces acting on it (the system). A failure then is linked to the interaction between the event and the characteristics which differentiate one event from the next, and the system and the characteristics which differentiate one system from the next. This paper focuses on sustainability and system continuity in terms of understanding conditions that promote system failure from the perspective of the event characteristics. It examines threats to system failure in the context of the characteristics of the event which causes the disturbance threatening system stability.
\end{abstract}

Keywords: Sustainable Development, System Failure, System Stability, Disturbance, Event Characteristics

\section{Introduction}

While performance along social, environment and economic dimensions has gained traction in business and society $[1,2,9]$, the focus has been on system continuity $[7,19]$. Because of the different interpretations which can be attached to sustainability or sustainable development [15], consensus on appropriate goals of the system, whatever the system might be, may be more difficult to develop than consensus on what is not wanted - such as a system failure.

System failure in this context is meant to convey a system's inability to adapt to changes in the external or internal environments brought on by an event that disturbs the equilibrium of forces acting on it (the system). A failure then is linked to the interaction between the event and the characteristics which differentiate one event from the next, and the system and the characteristics which differentiate one system from the next.

This paper focuses on sustainability and system continuity in terms of understanding conditions that promote system failure from the perspective of the event characteristics. It examines threats to system failure in the context of the characteristics of the event which causes the disturbance threatening system stability.

The interaction among sustainable development, sustainability, and system failure is presented in section 2 . It does so in the context of Figure 1 which places a system in terms of two states, one in which the system is functioning normally, able to adapt to disturbances without threatening system integrity. The second state is where disturbances lie outside of the system's ability to respond and so invoking the system's problem solving routine. Disturbances to the system are then caused by events.

Section 3 focusses on the event causing a disturbance that the system may need to respond to in order to ensure system continuity. These events then have characteristics, or in other words, attributes, which can influence the ability of the system to respond, restoring the old system, adapting itself to a new external and internal environment and so avoiding failure, or suffering system failure. The response process is examined through events each possessing one of seven characteristics. Each of the events is then placed in the context of a scenario to better illustrate the impact which the 
characteristic can have on system failure. In addition, the influence which different characteristic states has on chance of system failure is examined through selection of events that possess two characteristics with each characteristics having two possible states.

Section 4. captures some key conclusions from the preceding discussion and provides some avenues for future research.

\section{Sustainable Development, Sustainability, and System Failure}

Every generation, every society, every organization, family, person or even idea is faced with challenges which need to be overcome or contained if it wishes to prosper and grow (sustainable development), maintain relevance (sustainability), or simply survive (avoid a system failure). Whatever the level or complexity, the system under scrutiny is itself made up of components, performing specialized tasks necessary to avoid system failure.

These components together reconfigure inputs in the process of generating the output of the given system. Components can be thought of as unable to survive in the absence of other components in the system withdrawing the assumption of redundancy. Survival then of the system is linked to survival of the components.

The system over time reaches equilibrium with its internal and external environments in such a way that system continuity is not threatened. Output in aggregate of a system tends to be stable in the absence of changes in the external and internal environments. Operations are conducted according to commonly accepted routines with autonomic adjustment mechanisms built into the system to return the system towards its goal state whenever deviations within the system between actual and desired levels pass a certain threshold level.

Disturbances not causing deep damage to fundamental parts of the system (such as an increase of unemployment within a macroeconomic model) are dealt within the context of autonomous routines (increase in transfer payments through higher unemployment insurance disbursements) that do not alter relationships among components (such as sectors in the macroeconomics model or stakeholders in the business model) and how the components are linked. Components are happy with the allocation of the output, with the output produced and how the output is produced. Components are for the most part willing participants of the total system. Disturbances that are linked to the three questions of where, what and how are resolved in the context of the system, its existing hierarchal relations, and decision making paradigms, and so restoring the system to a state that does not threaten system continuity.

Disturbances that pose a threat to the existing system structure and the relationships among the system parts are different such as when a quorum of components become disenfranchised with the attributes of the system. The functioning of the components and interaction among the components no longer can be taken for granted, with the system structure, hierarchical relations and linkages among system components being in dispute. Deviations beyond some threshold levels result in a search process for solutions on how the system can adapt to the changing environment. The decision maker(s) do(es) not know how to proceed from the given state to the desired state and need(s) to embark on a problem solving routine.

This process can result in a paradigm shift in how the system is structured in terms of linkages and/or hierarchy, in the value set which is used to allocate resources within the system, in the goals to which the system aspires, or restoration of the old regime governing system operation. If a solution is not found to the present problem, here taken to be disturbance levels threatening system continuity, there are consequences or costs that must be born either by the decision maker(s), by the component(s) to which the decision maker(s) belong(s), to other components, or to the system as a whole.

Figure 1 illustrates the disturbance process showing time along the $\mathrm{x}$-axis and degree of disturbance along the $\mathrm{y}$-axis. The horizontal line intersecting point " $A$ " on the $y$-axis represents the maximum disturbance (caused by some event), that the system can tolerate with its (the system's) characteristics without embarking on the problem solving routine. Solutions to disturbances lying below "A" can be handled within the context of autonomic routines that restore equilibrium without the need to initiate new search routines. The system knows how to address the current problem. System hegemony is not threatened.

Once disturbance levels rise above this line however, the system is unsure how to proceed. Its authority is in question, but it does not know how to respond. Should it view these disturbances as caused by rogue components, not representing mainstream views or behavior and thus need to be contained or eliminated? Or should it view the disturbance as an indication of fundamental change in behavior brought on by some systemic changes that require adaptation of existing attributes of the system to meet the new realities?

Events leading to disturbances falling below line "A" are not of interest. These can be addressed within the context of the existing system and do not represent a threat to system continuity. What is of interest are events which cause an increase in the disturbance levels above, or some series of events leading to a continual rise in disturbance levels above, "A".

\footnotetext{
${ }^{1}$ Disturbance levels above "A" will lead to one of four outcomes. There will be: (1) a system failure because of an inability to quell the disturbance; (2) a paradigm shift in the existing system allowing for continuity from one system the next; (3) restoration of the old regime; or (4) in quelling the current disturbance changes to some characteristics of the existing system which will make system failure more likely in the next period or when the system experiences the next above " $A$ " disturbance causing event. With the focus on system failure, interest is in " 1 " and "4" though " 4 " here.
} 


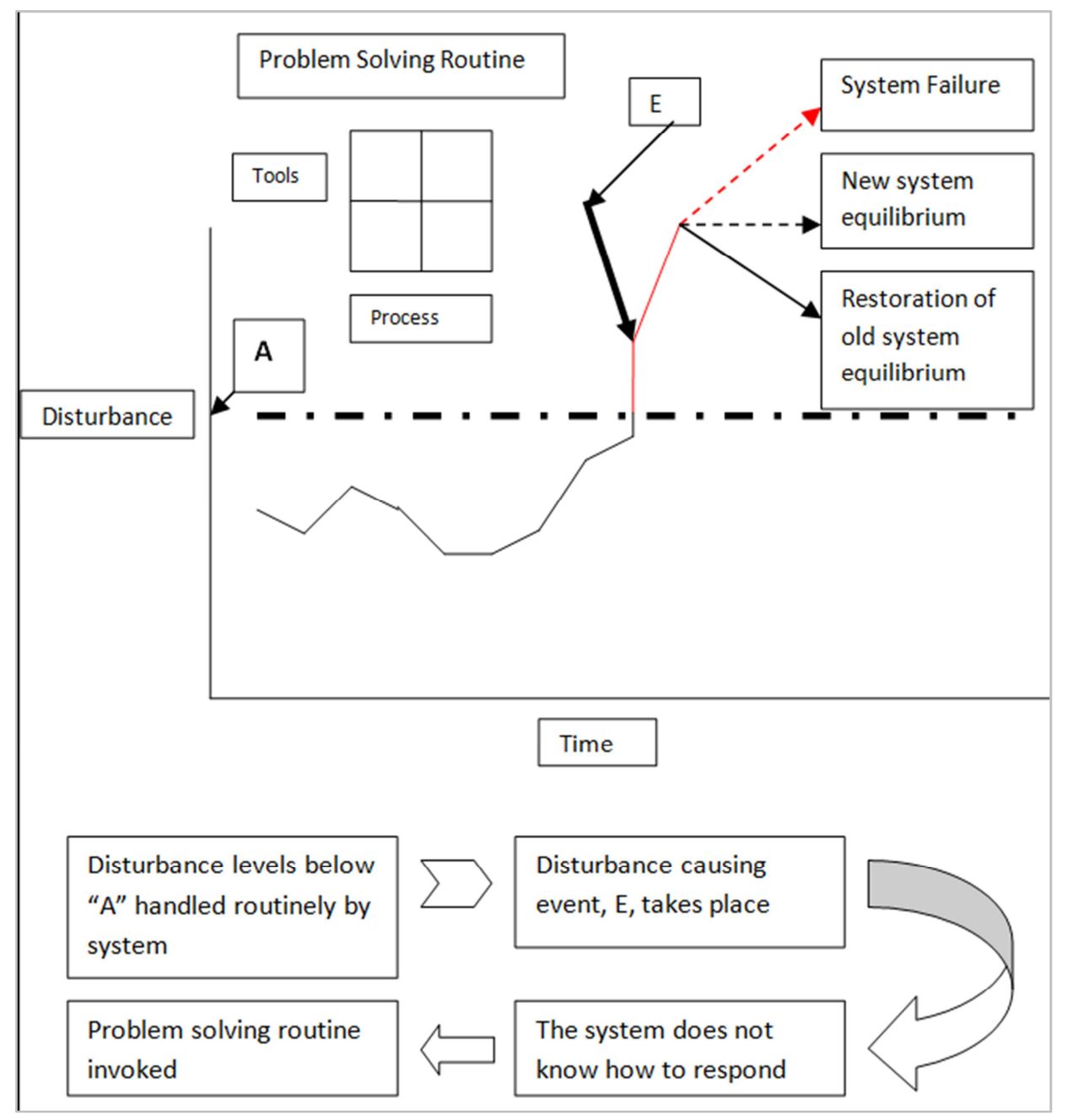

Figure 1. Disturbance levels and system failure.

\section{Event Characteristics}

The event cannot be separated from the system when investigating the risk of a system failure. For one system, the event may not pose a threat to continuity because of its (the system's) better resources and greater capacity to absorb the disturbance (without invoking the problem solving routine). Another system because it lacks the ability to identify and implement a solution to the present crisis (caused by an event) could suffer a system failure. Thus to understand system failure and the risks linked to its occurrence both the event characteristics and the system attributes, including attributes which affect the problem solving routine, must be investigated. However, as a first step to unbundled the interaction between event and system, this paper focusses on how characteristics of the event might impact on the chance of system failure independent of the system characteristics/attributes.

The chance of system failure stemming from an event is linked to seven characteristics. To demonstrate the lack of independency among the characteristics, two interactions are illustrated. The interactions are inserted immediately following the two characteristics which interact, labeling the interactions as characteristics " 5 " and " 9 ". The characteristics are: (1) event type; (2) frequency of event; (3) scope of event; (4) severity of event; (5) scope and severity interaction; (6) complexity of event; (7) actual time to event; (8) perceived time to event; and (9) actual time and perceived time interaction.

\subsection{Type of Event}

In order to qualify for an event in the context of this discussion, it must be an event that is different from what is normally expected given the existing state of the system. An event that is an expected event will have limited impact on the current state of the system. The system will have already adjusted to its anticipated consequences notwithstanding that some component(s) of the system, no matter how sure it is or they are that an event will occur, will still deny its arrival and its ensuing consequences. ${ }^{2}$ In order for the event to have an impact, it must be sufficiently different from a normally expected event in order to trigger the search process for a

\footnotetext{
${ }^{2}$ Here it is assumed that components, or at least some of them, are sentient.
} 
solution. The search process is initiated because the disturbance levels have risen above " $A$ " in Figure 1. The system continuity, hegemony, or both is threatened, and it (the system, its decision maker(s), or both) do(es) not know what to do.

This concept can be illustrated in terms of a transportation system consisting of an interconnected network of surface roads as one component and an accompanying component of ditches to remove moisture from the system. A decision maker component is not present. The capacity of the drainage structure is linked to the expected volume of moisture falling in a given time period. As long as the quantity of moisture falling (the event) remains within the quantity of moisture the structure was designed for, the chance of a failure of the overall system is not high. Here failure is defined as the unacceptable decrease in capacity of the network to handle traffic. If however the system is subjected to an unanticipated high volume of moisture in a short time period, the event, which exceeds the capacity of the drainage component to handle, there is a chance that the overall transportation system will fail, either in terms of decreasing the capacity to support movement of goods per unit time below some threshold or an outright failure of the system to facilitate the movement of goods per unit time.

In this scenario, the normal range of the event is a distribution of rainfall over a given time period. As long as the total rainfall is less than the maximum volume within the normal range, the transportation system is not likely to fail (from a moisture perspective). As soon as the amount of rainfall lies outside the maximum volume, the chance of a failure or partial failure increases. In this case, the higher than normal volume per unit time, the characteristic of the event, is above the maximum in the range, and thus further the event from the expected norm.

This leads to the first hypothesis.

H1: The greater the distance between the event that has occurred, and what are considered events of normal occurrence, the greater the chance of a system failure.

\subsection{Frequency of Event}

A solitary occurrence of an event is not necessarily sufficient to overcome the inertia of the incumbent system conditions. In other words, one occurrence may not be sufficient to maintain disturbance levels above the threshold line in Figure 1 and so invoke the search routine even if the initial event led to disturbances momentarily being above "A". There may be a fall off in observed disturbance linked to a particular event over time as the autonomic healing process of the system kicks in. In other words, the ability of the event to keep disturbance levels above " $\mathrm{A}$ " is time dependent. Thus in order for an event to have an impact on the chance of a system failure, the frequency of events must also be considered, holding constant other event characteristics.

This concept can be illustrated through the cumulative impacts of snow storms on the ability of a local municipality to keep their transportation system functioning, i.e. facilitating the flow of goods and services throughout the city. In this scenario, the system consists of a road network as a component, a snow storage facility as another component, a snow removal component consisting of a number of trucks and shovels to remove snow in solid form from the road network to the storage network, and snow removal component from the storage component based on a migration of water in solid form (snow) to liquid form (water) through temperature variations in the overall system (the autonomic recovery process built into the system). The number of trucks and shovels defines the capacity per unit time to remove snow from the road network. Temperature days above some threshold defines the snow removal capacity for the storage component. A decision maker is not present in this example. System failure is defined as a sharp reduction in the capacity to transport goods and services within or across the city limits.

The system in this circumstance is the transportation system. There is limited capacity to locate the snow removed from the city streets, which here assumes that the capacity of the snow removal from the street component is not exceeded. As the first snow storm comes, the storage capacity begins to fill up. However, after the first snow fall (the event), there is ample space left. The second snow fall comes. The accumulation of the first snow storm has not melted away, the autonomic healing process of the system to restore system capability. After the consequences of the second snow storm have been removed, there is even less storage capacity left. This sequence continues until all of the capacity is used and there is no storage space left. The system, in this case the road transportation system in the municipality, may fail. The next snowfall might lead to a paralysis of the transportation system since there is insufficient place to put the snow (if the autonomic recovery process does not generate the required space in the time frame required).

The occurrence of one event, snowfall, does not lead to long-term paralysis of the transportation system putting aside the short-term paralysis that of course can occur when capacity of the trucks and shovels to remove snow within a given time period is exceeded. The snow clearing component has sufficient capacity to move the snow from the road network and sufficient capacity to store the snow in the storage component. In the presence of temperatures above freezing in the time period separating successive snow falls, accumulated snow departs from the system in a way that does not impact on the capacity of the transportation system, i.e. the drainage component is adequate to the task. However, the occurrence of another snowfall before the accumulated snow in the storage location has melted away increases the share of the storage capacity utilized. As this utilization level increases so too does the chance that the next event, a snow fall, will more than fill-up the remaining capacity. When this occurs, the ability of the system to perform its role - facilitate the movement of goods within the municipality - can be sharply reduced or completely stopped.

This leads to the following hypothesis.

H2: The higher the frequency of the event, the greater the 


\section{chance of a system failure.}

\subsection{Scope of Event}

In addition to the non normality of the event and the frequency of the event occurrence (whether normal or not normal), the scope of the consequences of the event on the system must also be considered. If only a small part of the system is affected by the event, the resources necessary to restore equilibrium could still be available, surplus to those necessary for basic maintenance of the unaffected parts of the system. However, as a greater proportion of the total system is touched by the event, the absolute amount of resources surplus to basic maintenance of the unaffected parts of the system decreases. Remembering that the resources allocated for restoration are unavailable for basic system maintenance of the unaffected parts of the system, the healing capacity of the overall system is thus reduced. Here some form of redundancy is present, i.e. withdrawal of some component(s) do(es) not immediately result in system failure.

This concept can be illustrated by considering a flood, the event, on a city, the system. The system consists of a number of components providing services and a number of districts providing geographic division. Components include public safety, food and water distribution, waste removal, basic transportation and health for example. The components are distributed evenly throughout the system such that survivability of the system is not immediately threatened by failure elsewhere in the system. Each district has the appropriate distribution of components to ensure continuity independent of other districts within the system, and further, that each district has the ability to produce a surplus of resources to that necessary for district maintenance. System failure is defined as the inability to provide basic services to support a civil society. A time element is also introduced such that restoration of service capabilities must take place within a certain period of time otherwise service capability is permanently lost.

A flood (the event) inundates the city, the system, and impacts a number of districts in the city. Those districts which the flood touches are assumed no longer to have the capability to provide basic services. The flood recedes but the capability of the districts touched by the flood to provide basic services is impeded. Restoration of service rests with resources made available from unaffected parts of the system. If a small number of districts relative to the total are touched by the flood, then there would most likely be sufficient surplus resources made available from the unaffected districts of the city within the time frame available to restore affected districts to pre flood state. However, as an increasing number of districts are touched, the minimum resources necessary to restore capability increases while the amount of resource surplus to the needs of the untouched districts that can be allocated to system restoration decreases. The chance of a system failure correspondingly increases.

This leads us to the third hypothesis.

H3: The narrower the scope of event relative to the size of the system, the lower the chance of a system failure.

\subsection{Severity of Event}

While the normality of the event, an event's frequency and its scope are important considerations, the severity of the consequences of the event on the system must also be considered. With severity, the extent to which the event impacts on the system performance is considered, and brings up the concept of tolerance and the capacity of the system to adjust to the event.

A system can have a range of states in variables representing the external environment (which is outside of the system's ability to control) over which system continuity is not threatened though performance is. Over this range of states, the system adapts to the new external conditions while performance may suffer. This (range of states) is the tolerant range of the system. If the value of the variables change outside of the tolerant range of the system, then system failure ensues. Severity then we define as the extent to which the consequences of the event lead to changes which impact system performance through its (the system's) ability to adapt to changing states of the external environment.

These concepts are illustrated with the following simplified system. In order to demonstrate adaptation and failure two self contained subsystems within an overarching system containing both are included. The sub-systems are labelled as "I" and "II". The influences from the external environment are reduced to one variable. The sub-system can reconfigure inputs over a range of states of this one variable. Assume that the range of possible states for this one variable is from " $\mathrm{A}$ " to " $\mathrm{Z}$ " inclusive. However, the sub-systems can only operate at or above maintenance levels for a subset of these states. Outside of the subset continuity of each sub system let alone the total system is threatened. The range of states over which the system can operate at least at the maintenance level is called the tolerance range. While the system can maintain activity over this range, performance levels vary across the sub-set of states. For sake of illustration, assume that the tolerance range for sub-system "I" is states "J" through "N". Peak performance occurs at state "L" while maintenance level performance occurs at the other four states. Sub-system "II" has a different tolerant range which overlaps with that of sub-system "II". The tolerant range of "II" is "N" through "P" with peak performance taking place at "O". Maintenance level performance occurs at the other two states.

Initially, the system is at equilibrium with sub-system "I" dominating the space. A small immeasurable portion of the space is occupied by sub-system "II". The state of the variable is "L" which lies outside the tolerance range of "II". An event takes place which causes the state of the variable to change from "L" to "O", the state "O" lying outside the tolerant range of sub-system "I" but lying in the tolerant range of "II". This leads to a change in the share of the space of the total system occupied by each sub-system in favor of sub-system "II". The event had high severity leading to a failure of one of the sub-systems, that of "I". If the impact of the event had resulted in a change of state from " $L$ " to say 
"M", severity would have been lower and would not have resulted in sub-system failure.

To bring the discussion into the real world, substitute global warming as the event. Now define the system as a climax plant community which has reached equilibrium with the physical environment including variations which exist daily and seasonally. A system failure in this context is defined to be a major change in the climax vegetation community. Now, introduce the event, global warming. The system, an existing plant community, cannot isolate itself from the event, a rise in temperature. The change directly touches all members of the community, transmitted through the medium of the air. The scope is thus high.

Providing the increase in temperature is small relative to the range of temperatures that the plant community can tolerate and still remain the climax vegetation cover, then the severity of impact on the system, the existing community, is low. A low severity event such as a small rise in temperature that falls within the range of the system to tolerate regardless of scope and frequency, may not lead to system failure. Some members of the community may be under stress and on the margins replaced, but overall the community remains generally intact. On the other hand, if the change in temperature is large, putting under stress the existing plant community, with a different plant community under the new temperature regime having a competitive advantage over the existing one, then the severity of the event is high. The system, in this case the designated plant community, is unlikely to continue as the climax community.

This leads to the fourth hypothesis.

H4: The greater the severity of the event on the system the greater the chance of a system failure.

\subsection{Scope and Severity Interaction of Event}

It is clear from characteristics " 3 " and " 4 ", there is an interaction between scope of event and severity of event. The scope identifies the percent of the total system under scrutiny which is touched by the event and its consequences. A system or portion of a system may be touched by the event but have a variable impact on the ability of the system to reconfigure inputs into its output, in other words, its performance. The impact on performance could be minimal, i.e. no measurable change in output or inputs, or it could be maximum, system ceases to generate output regardless of the availability of inputs, and performance falls to zero. In the first instance, severity would be low. In the second, high.

To understand the interaction between scope and severity of event on the chance of a system failure, take two possible states for each of the characteristics, low or high. Now form a two by two matrix with scope along the y axis and severity along the $\mathrm{x}$ axis. There are then four possible quadrants as shown in Figure 2. Quadrant One is the intersection of low severity and low scope. Quadrant Two is the intersection of low severity and high scope. Quadrant Three is the intersection of high severity and high scope. Quadrant Four is the intersection of high severity and low scope. The objective is to rank the quadrants in terms of the chance of a system failure, holding constant the system.

To better illustrate the interaction, events from the natural environment impacting on a given system is used. The system is a city. In other words, for the four events selected, the system is constant. What changes (other than the event) is the chance that the city, the system, will not recover from the event and its consequences, leading to a system failure. System failure is defined as for characteristic " 3 " as the inability to provide for basic services of a civil society. Events with characteristics of each of the four quadrants are a large scale meteor strike, a flood, an extended period of higher temperatures and humidity, and a localized hail storm. Restrictions as identified are introduced to ensure that events conform to the quadrant characteristics into which they (the events) are placed. All events are assumed to be abnormal for the system.

In Quadrant One, severity is low and scope is low. Think of a localized hail storm that only touches a small portion of the districts in the city. The event does not disrupt services for extended periods of time within the districts touched by the storm. Disruption is at most momentary. While some damage takes place, and some services can be interrupted during the storm, the resources necessary to restore pre-event conditions are not large and can most likely be provided by the districts affected themselves. A chance of failure here is the lowest of the four quadrants.

In Quadrant Two, severity is low and scope is high. An event that falls in this category would be abnormally high temperatures and humidity over an extended period of time, the abnormality of which has a natural ending linked to seasons. All parts of the system are touched excluding the scenario in which temperature and humidity can be controlled within parts of this system. The scope is thus high. However, the abnormally high temperatures/humidity are not out of the tolerant range of the system, its physical and biological components. While the severity is low, the performance levels of the system are decreased because the temperature and humidity to which the system is subjected has deviated from optimal levels. The capacity of the system to generate surplus resources has decreased. The chance of a system failure is seen higher than with Quadrant One due to a decrease in surplus resources available to avert future system failures and closer proximity of the system to the temperature and humidity beyond which system failure occurs. The closer the current temperature and humidity are to the boundaries of the tolerant range, the greater the chance that another incremental change will push the system outside its limits for operability. There is less room to absorb the increase or decrease depending on which end of the tolerant range the system is. A medium chance of system failure is assigned.

In Quadrant Three, the scope and severity are both high relative to the system, a city and its ability to provide services in support of a civil society. The event is an impact of a large meteor close to the city. The basic environment is altered completely denying existing flora and fauna basic ingredients to support continuation of existing associations. Recovery is not immediate. Remedial activities on the part of system to 
restore the city to preexisting conditions are not possible as ability of the system to generate resources surplus to maintenance levels has been severely impeded if not completely disrupted. Here assume that the city was located close to or at ground zero of the event. The city cannot isolate itself from the event or its consequences. All districts are affected. The scope is thus high. Not only are all districts of the city touched by the event (and/or its consequences), but the capability of each district to maintain even basic services such as water (in support of a civil society) is destroyed. Severity is thus high. Without intervention from outside the system, the likelihood of a system failure is high to extreme, the highest of all four quadrants.

Now focus on Quadrant Four, high severity but low scope, and modify slightly the system, to include a series of interlocking dikes designed to limit the spread of a flood, the event. Following the discussion presented in characteristic " 4 ", limit the flood to a small portion of the city, say less than 10 percent of the districts. Any district which the flood touches sends its (the district's) performance to zero with zero chance of recovery without surplus resources made available from the untouched districts. Even with the flood receding, performance does not change for the touched districts. For those affected districts, severity is high. However, since only a small share of the total districts were touched, the scope is low. The ability of the untouched districts to restore the touched districts to pre-event condition is good providing surplus to maintenance level resources exist. The event for the most part does not destroy the infrastructure supporting the delivery of basic services. It simply disrupts the pathways which connect the different components together. Restoration is easier than in the case of the meteor for which pathways and the infrastructure are assumed heavily damaged. Thus chance of failure in Quadrant Four is less than Quadrant Three. Chance of system failure for this quadrant is assumed medium.

An interaction affect between scope and severity using Quadrant One versus Quadrant Two and Quadrant Three versus Quadrant Four has been demonstrated. However, there is insufficient information to unambiguously differentiate Two from Four.

This then leads to the following hypothesis.

H5: The chance of a system failure will be highest to lowest with event characteristics as event (severity, scope): $(H, H)>(H, L)=(L, H)>(L, L)$.

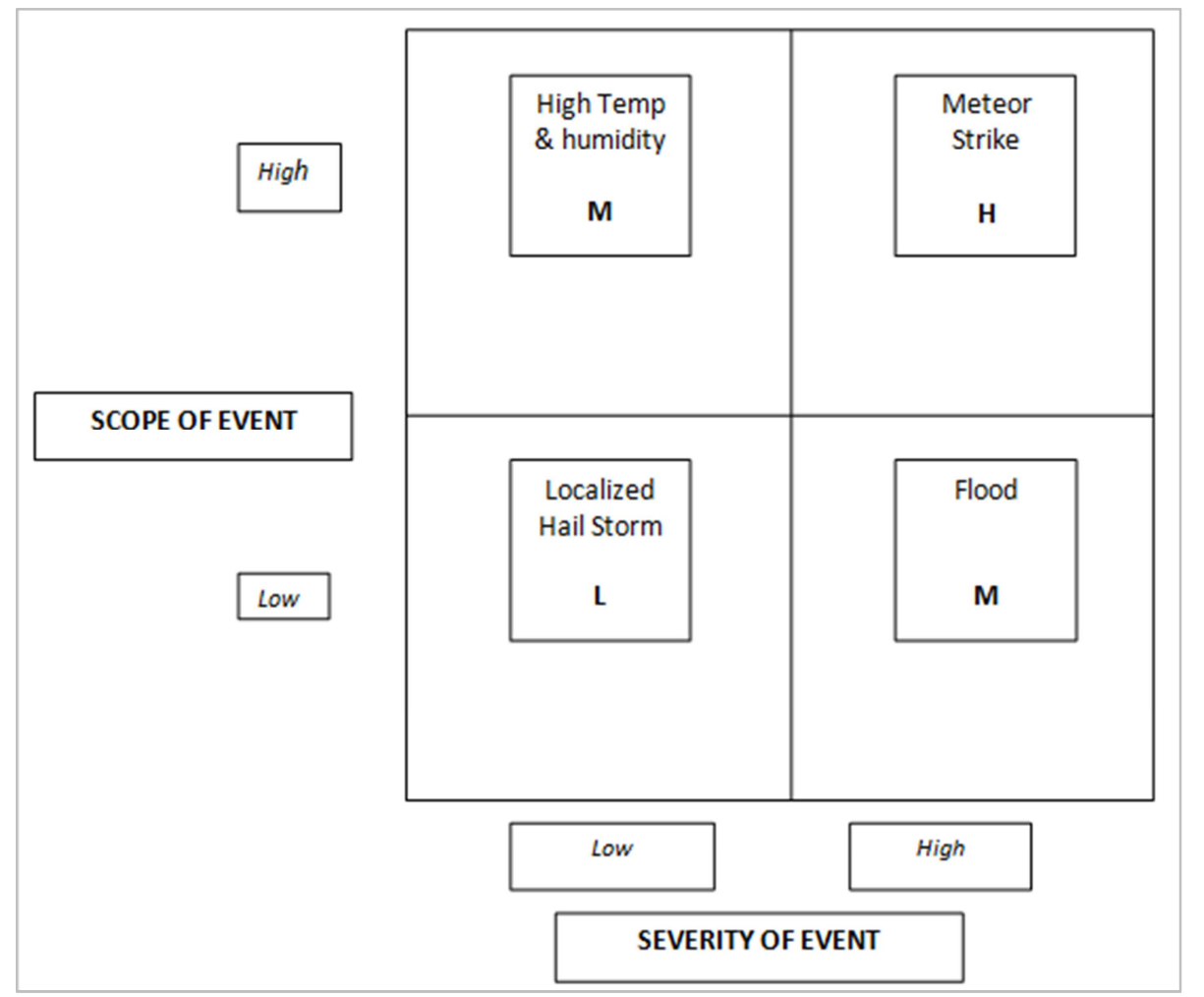

Figure 2. Interaction between scope and severity of event on chance of a system failure with the designated event.

\subsection{Complexity of Event}

The previous discussion has shown that event type, its frequency, scope and severity are relevant. Complexity of the event is also an important consideration.

Neither the root cause of the event nor its full consequences may be clearly understood. It is the unknowingness of the impact of the event or the event's root causes, even when all characteristics of the event are understood, that represents the threat to sustainability of the system $[16,14]$.

These dimensions are illustrated through the following scenario (adapted from [12]), first focusing on the unknowingness of the root causes of the event. Define the system as a medium sized body of water connecting a 
number of separate land masses. Also incorporate subsurface geology under the water. Include a floating drill platform the purpose of which is to drill for oil under the water. A floating platform drilling an exploratory oil well off the coast of one land mass in deep water suffers a catastrophic failure leading to destruction of the drilling platform, the event. The event leads to a rupture of the well head deep underwater, the first order consequence. The second order consequence is the ensuing discharge of oil into the surrounding water linked to the ruptured well head. The causes of the event, destruction of the platform, and the ensuing consequences, may be multiple and ambiguous, thus interfering with activities designed to prevent the occurrence of a similar event. Is the technology used at fault, i.e. is it incapable of handling the array of conditions normally expected in the operating environment? Change the technology, and change the outcome. Perhaps the technology itself is not at fault, rather it is the quality of the particular pieces of equipment using that technology that was the root cause. Improve quality control of the manufacturer of the equipment and change the outcome. Rather than the technology itself as the culprit, it is the application of the technology by the operators that is flawed in terms of the trade-offs among safety, economics and the environment. Change the operator, and change the outcome. Rather than the individual operator as the root cause, it could be the standard practices of the industry itself where the root cause lies. Changing the operator would not change the outcome everything else remaining the same; but changing the standard practices would. It could be linked to unique features of the geology surrounding the well. Everything was done to the best standard with respect to the well, but unique and unanticipated geology connected with the oil bearing rock resulted in tolerance levels of the existing system being exceeded. It could of course be more fundamental. A society wedded to petroleum based energy which is becoming harder and more difficult to develop in a riskless way. Reduce the need for petroleum based energy reduces the need to go into environments in which regardless of what is done and how it is done, accidents happen.

Limited resources and multiple possible causes, the solutions to which cumulatively exceed the available resources increases the chance that a second event may happen. While some of the causes can be addressed, it is far from certain if these are the root causes of the current event let alone the most important causes of some future events.

Turning now to the second dimension. If the original event has not happened before or the event has happened but the consequences were limited, or there was misplaced confidence on the capacity of the system to contain the initial consequences of the event because of past experience in similar situations though different environments, the uniqueness may pose threats because of uncertainty of consequences. In other words, there is a lower sense of urgency than is warranted which influences the priority attached to the use of available resources in a negative way.

Returning to the initial event and then turning to its second order consequences, the impact is initially localized in terms of scope, i.e. geography, and in terms of severity, small concentrations of the hostile substance (hydrocarbons) not overwhelming the restorative capacity of the system under scrutiny. However, as the initial source of contamination continues unabated, it cannot be completely contained. Tools, techniques, and approaches which were thought adequate to the task were not successful. The initial equilibrium of flora and fauna in the system is disturbed and it is unclear what the new equilibrium will be and in turn what the secondary impacts of the new equilibrium, whatever that might be, will have elsewhere in the system. It may take years for the consequences of the initial event to dissipate with unknown consequences because of the interconnectivity of systems and subsystems. If a second occurrence of the event takes place notwithstanding the restorative capacity of the system, the tolerance limits can then be exceeded on some components with unanticipated consequences.

Complexity then leads to our sixth hypothesis.

H6: The greater the complexity of the event the greater the chance of a system failure.

\subsection{Time to Event}

Time to event is a factor that is linked to the system and its ability to orchestrate and then deploy the resources necessary to neutralize the consequences of an event. Here assume that the root causes of the event have been identified and a solution to the event and its consequences are known and required resources for problem solving have been dedicated. It is just a matter of ensuring adequate resources are deployed. The longer the lead time the larger the amount of resources that can be allocated to address the event and its consequences. The larger the resources made available the lower the chance of system failure everything else being the same.

This is illustrated through a simple paradigm of a system with a maximum fixed capacity to deliver relief supplies to victims of a natural disaster, say a flood. Here assume that the inventory component at the site of the disaster is not a limiting factor nor the time that the supplies are in inventory before distribution. In other words, the storage capacity at the disaster site is sufficiently large to handle the maximum quantity of supplies possible and there is not spoilage of the supplies in inventory. A system failure in the disaster area will happen if a minimum quantity of supplies fails to arrive by a certain time. The maximum quantity that can arrive by the time is determined by the lead time, in this case the time to the event. The larger the lead time, the greater the quantity of supplies which can be accumulated at the disaster site, and thus a lower likelihood that a system failure will occur (due to the quantity of supplies available at the disaster site).

Now, transform this illustration slightly and place it into a probabilistic frame. When the event will occur, say a flood, is known, but what is not known is the quantity of supplies that must be on hand to avert the disaster, say a large scale famine, the consequence, connected to the event. What is known from past experience however is that the quantity of 
supplies that must be on hand for any given event (a flood) follows say a normal distribution. Importantly, the event in this transformation is the flood and not the insufficient quantity of supplies. A system failure occurs if the consequences of the event which is taking place at a known time leads to a quantity of supplies required which exceeds the capabilities of the delivery system in place. It could be less than the scenario illustrated earlier or more than this. It is just that a priori what is not known is the quantity of supplies that is needed. Thus the greater the lead time the greater the quantity of supplies which can be deployed and so a wider range of possible quantities out of the total of all quantities that can be made necessary by the event (a flood) that can be met. Accordingly there is a decrease in the chance of system failure.

This thus leads to the seventh hypothesis:

H7: The greater the time until the known event takes place the lower the chance of a system failure.

\subsection{Perceived Time to Event}

Allocation of resources in the context of a system involving scarce resources brings in the need for a paradigm to allocate the required resources among competing uses. If there is no urgency attached to the problem under scrutiny, and there are competing uses for the limited resources that can be deployed to address it (the event and its consequences), little can be expected in the way of progress towards its resolution, and thus averting a system failure howsoever defined. Resources have not been made available either because other uses have out competed for them, or the decision maker(s) has (have) not yet made a decision on which uses for the available resources.

As the perceived time until the event takes place diminishes, then urgency would increase with a corresponding increase in resources deployable to solve the problem everything else being the same. In other words, in the pool of all possible uses for the available resources (consequences of events or otherwise), the particular use gets a higher priority and preferential access to them (the resources). Importantly, the actual time to the event is held constant. Here assume that the actual time to the event has no impact on the chance of a system failure and that there is a one time allocation of resources. In other words, as long as the resources are deployed, it does not matter when they are as long as they are deployed before the event.

This then leads to the eighth hypothesis.

H8: The shorter the perceived time to the event the less the chance of a system failure.

\subsection{Perceived Time to Event and Actual Time to Event Interaction}

However, it is not only the perceived time to a system failure causing event that is important. It is also the actual time until the event takes place that is important, thus relaxing the restriction placed immediately above.

If perceived time until event is long, but the actual time to event is short, the system howsoever defined will have been unable to adequately prepare for the event or its consequences. The capabilities of the system can be overwhelmed thus leading to a system failure. It follows that the chance of a system failure increases due to inadequate preparation. Correspondingly, where the actual time to the event is far in the future, but the perceived time to the event is near, there is a smaller chance of system failure. Adequate resources are dedicated to finding and then executing on a solution. A long lead time available for the dedicated resources to have an effect would diminish the chances for a system failure to take place. Thus there is a lower chance of system failure.

This is illustrated through Figure 3 which shows two time lines, one for the actual time until the event takes place and the other for the perceived time until the event takes place. The actual time in the future when the event takes place is identified as point " $E_{\mathrm{A}}$ ". For illustrative purposes assign a value of 50. Thus the event will take place 50 time units hence. The perceived time line identifies the time until the event takes place from the decision maker(s) viewpoint. Labeled are three times as $\mathrm{E}_{\mathrm{P} 1}, \mathrm{E}_{\mathrm{P} 2}$ and $\mathrm{E}_{\mathrm{P} 3}$. $\mathrm{E}_{\mathrm{P} 1}$ corresponds to an event that is perceived to happen shortly. For illustrative purposes assigne a value of 10 time units. $\mathrm{E}_{\mathrm{P} 2}$ corresponds to the perception that the event will take place slightly further into the future, in the illustration identified as 40. The final perceived time is assumed to be even further into future and is assigned a value of 75 . Notice that $E_{P 1}$ and $\mathrm{E}_{\mathrm{P} 2}$ both are shorter in duration than when the actual event takes place. $\mathrm{E}_{\mathrm{P} 3}$ on the other hand takes place well into the future, beyond when the actual event takes place.

With this figure, three scenarios are illustrated. The first scenario is when the perceived time very much precedes the actual time when the event occurs. In this scenario the difference between $E_{A}$ and $E_{\mathrm{P} 1}$ is quite large, as in Figure 3 where there is a value of 40 . In the second scenario, the perceived time to the event is closer to the actual time of the event but is still less than the actual time. Thus the difference between the actual and perceived times, while still positive, is less than with the first scenario. The third scenario is different than the first two in that the perceived time to the event is greater than the actual time. In our illustration, $\mathrm{E}_{\mathrm{P} 3}$ is very much greater than $\mathrm{E}_{\mathrm{A}}$. Thus the difference between the actual time and the perceived time is negative.

Take Scenario 1. The perceived time until the event is in the very short term (relative to when the actual event takes place). The short term then helps prioritize resources for allocation to the designated problem among all the alternative problems/issues to which they could be allocated. Allocating the resources sooner means that there is a longer time period over which the resources can be deployed in order to address the event and its consequences. Everything else being the same, a long lead time results in a greater chance that either the event can be averted or the consequences of the event can be overcome, and thus avoid a system failure.

Turning to Scenario 2, the only attribute which has been changed is the perceived time until the event. Instead of 10 
time units until the event takes place, decision maker(s) now see the event happening in 40 time units. The actual event is still occurring 50 time units hence. The longer the time until the event takes place reduces the urgency which decision maker(s) assign to the event. The event then has a lower priority among all possible uses for the scarce resources. Resources are less likely to be forthcoming as soon. Depending on the quantity of resources necessary and the time required for the deployed resources to have the intended effect, the chance of a system failure increases.

The final scenario shows the disjoint between reality and perception of reality. The event will take place at a specified time in the future. However the decision maker(s) see the event in a different fashion. Because the event is perceived to take place much further into the future, the priority is much lower in terms of deserving resources. With a lack of resources, very little is done that directly impacts on dealing with the event or its consequences. While the consequences may not overwhelm the system, the system is not prepared until the event happens. Thus, the chance of a system failure is very much greater than when the perceived time is less than the actual time to the event, and there is correspondingly resources made available before the event actually takes place.

This leads to the following hypothesis.

H9: The greater the difference between actual time to the event less the perceived time to the event the lower the chance of system failure.

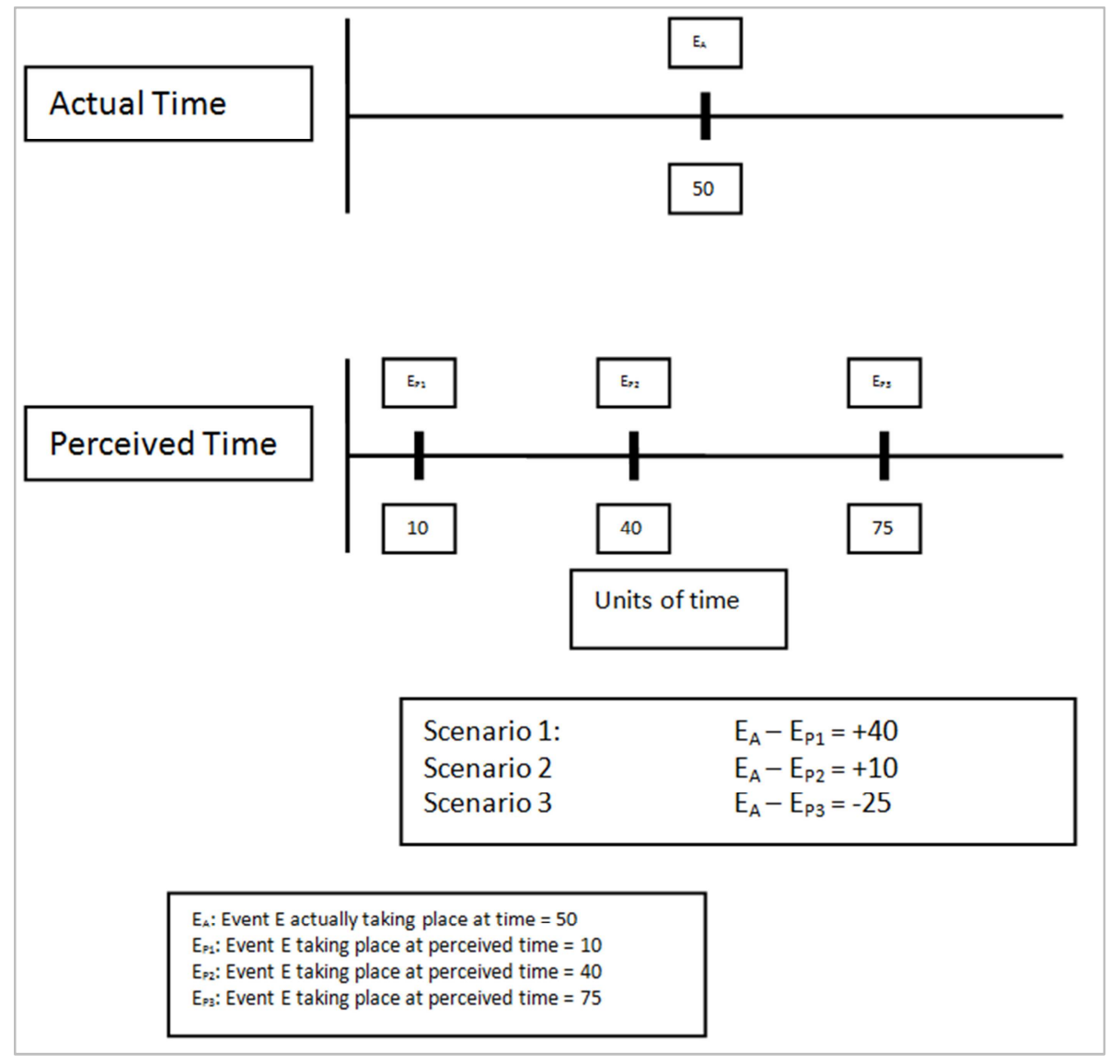

Figure 3. Actual time versus perceived time to event.

The interaction between actual time to the event and perceived time to the event is further illustrated using the two by two matrix concept introduced earlier. Along the $\mathrm{x}$ axis is shown the actual time to the event divided into two categories, short and long. Along the y axis the perceived time until the event is shown also divided into two categories, short and long. There are now four quadrants defined by the two by two matrix. Quadrant One is the intersection of short actual time and short perceived time. Quadrant Two is the intersection of short actual time and long perceived time. Quadrant Three is the intersection of long actual time and long perceived time. Quadrant Four is the intersection of long actual time and short perceived time.
The objective is to rank the quadrants in terms of chance of a system failure holding constant the event and holding constant the system.

The interaction is illustrated using global warming as the event. The commonly held view is that global warming has high scope and high severity though it is less commonly held when the actual tipping point will be reached. Through adjustments to population, technology and behavior many scientists believe that the crisis can be averted though there is a long lead time necessary before the remedies can have an effect. Global warming is linked to that increase in temperature beyond which the current system cannot continue in its current form. The system can be global 


\section{civilization.}

In Quadrant One, actual time to the event is short and the perceived time to event is short. With an actual time to the event as short, and assuming that the system has yet to respond, the room for missteps is small. If there is any controversy linked to prioritizing the resources for use in addressing global warming then the window to avert the failure is small relative to all possible outcomes. Here assume that there is still sufficient time to avert the disaster. A short perceived time allows for resources to be deployed because of the higher priority linked to their uses for the identified use. Time though in this quadrant is not an ally. Thus there is a high chance for system failure.

In Quadrant Two the worst of all scenarios - A short time to the actual event but a long perceived time in the decision maker(s). Resources are available in order to avert the catastrophe but the limiting factor is their orchestration to solve the problem. If the perceived time until the event is long into the future, then it is unlikely that the resources would be dedicated to addressing the problem before the event takes place. When the event takes place, it may be too late to avert the failure. Thus the interaction between a short time until the actual event takes place and a long perceived time suggests an extreme chance of failure.

Quadrant Three demonstrates a long time to the actual event and a long perceived time to the event. In the circumstance where the actual time to the event is long and the perceived time to the event is long there is less risk for a system failure. While the perceived time is still long, thus reducing the chance that resources could be allocated to averting the event, there is a greater opportunity that intervening developments could trigger a change in perception such that resources could be made available within the time frame to still avert the disaster. Thus, the share of outcomes out of all possible outcomes in terms of orchestrating resources which averts a disaster is greater than in the case of Quadrant One for which the actual time to the event is short and the perceived time is short. Thus assign a medium chance.

Quadrant Four demonstrated the most favorable outcome. If the actual time until the event takes place is a long ways into the future, this means that there can be sufficient time if remedies are put in place sooner for the consequences of the event to be averted. If the perceived time until the event is short then this results in the required resources and behavioral changes taking place within the time frame available to generate the cumulative effects. Thus the interaction between long actual time and short perceived time leads to a low probability of system failure.

This then leads to the following hypothesis as shown in Figure 4.

H10: The chance of a system failure will be highest to lowest with event characteristics having high scope and high severity as shown with event (actual time, perceived time):

$(S, L)>(S, S)>(L, L)>(L, S)$.

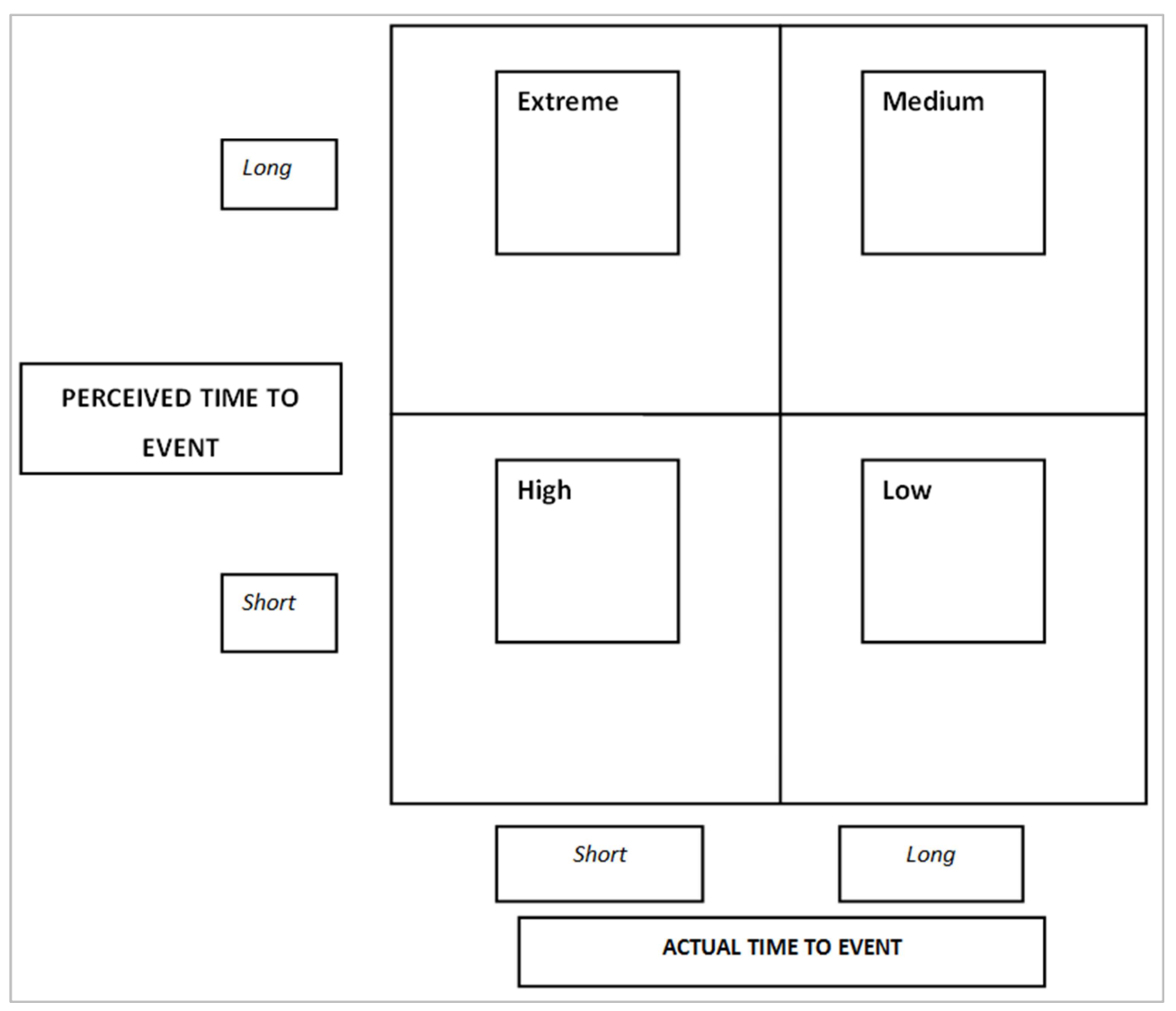

Figure 4. Interaction between actual time to event and perceived time to event having high severity and high scope. 


\section{Conclusions and Future Research}

The previous discussion has illustrated how event characteristics influence the chance of a system failure. Further, many of the characteristics of an event are not independent of each other in terms of their influence on the chance of a system failure.

It is also evident that system characteristics have an impact on whether an event can lead to a system failure or not. For example, define the event as the introduction of a hitherto unknown pathogen into a population, the extent to which a given population might be eliminated is based on the capabilities resident in the population to identify the root causes and then adapt itself to neutralize the negative effects. For example, if the population were humans ten thousand years ago, that population would not have had the capabilities in place to find a solution to avert the catastrophe. Go forward ten thousand years to the present day. The population has the capacity to identify the root causes and develop a strategy to avert the negative consequences on the population. For the first system, the chances of a system failure are high. For the second population, the chances are lower due to the resident knowledge.

To fully understand the chance of a system failure the interaction between the event and the system needs to be investigated. Characteristics of the event have been unbundled in this paper. The next step is to unbundle the system along a number of characteristics in the same way that was completed for the event. The final step is to link event characteristics with system characteristics to determine how the chance of a system failure varies.

\section{References}

[1] Bansal, P. and K. Roth. "Why companies go green: A model of ecological responsiveness." Academy of Management Journal 43, no. 4: p. 717-736.

[2] Buysse, K. and A. Verbeke. "Proactive environmental strategies: A stakeholder management perspective." Strategic Management Journal 24: p. 453-470.

[3] Commoner, Barry. The closing circle: nature, man, and technology. Knopf, 2014.

[4] Commoner, B. Making peace with the planet. New York, New York, Pantheon, 1975.

[5] Dodd, Dominic, and Ken Favaro. "Managing the right tension." Harvard Business Review 84, no. 12: p. 62-74.
[6] Ehrenfeld, John R. "The roots of sustainability: the real business case for sustainability requires more radical, fundamental and difficult change than most are ready to consider, but anything less ignores the real problem and may, in fact, contribute to it." MIT Sloan Management Review 46, no. 2: p. 23-26.

[7] Hall, Jeremy, and Harrie Vredenburg. "The challenges of innovating for sustainable development: To foster sustainable development, an innovation strategy must have vision that transcends a maelstrom of complex, and sometimes contradictory, demands." MIT Sloan Management Review 45, no. 1: 61-69.

[8] Hart, Stuart L. "Beyond greening: strategies for a sustainable world." Harvard Business Review 75, no.1: p. 66-77.

[9] Hart, Stuart L. "Worlds in collision." Journal of Organizational Excellence 25, no. 3: p. 13-25.

[10] Hart, S. L. and M. B. Milstein. "Creating sustainable value." Academy of Management Executive 17, no. 2: p. 56-67.

[11] Hawken, Paul, Amory B. Lovins, and L. Hunter Lovins. Natural capitalism: The next industrial revolution. Routledge, 2013.

[12] Ingersoll, Christina, Richard M. Locke, and Cate Reavis. "BP and the Deepwater Horizon Disaster of 2010." MIT Sloan School of Management, Case Study, 2012.

[13] Isenmann, R. "Industrial ecology: Its perspective of understanding nature as model." Sustainable Development 11: p. $143-158$.

[14] Jürgens, Hartmut, and Dietmar Saupe. Chaos and fractals: new frontiers of science. Springer New York, 2006.

[15] Keiner, Marco, ed. The future of sustainability. Amsterdam: Springer, 2006.

[16] Rosen, Robert. "Complexity and system descriptions." In Systems: Approaches, Theories, Applications, pp. 169-175. Springer, Dordrecht, 1977.

[17] Senge, Peter M., Benyamin B. Lichtenstein, Katrin Kaeufer, Hilary Bradbury, and John S. Carroll. "Collaborating for systemic change." MIT Sloan management review 48, no. 2: 44 .

[18] Shrivastava, P. "The role of corporations in achieving ecological sustainability." Academy of Management Journal 20: p. 936-960.

[19] Westley, F. and H. Vredenburg. "Sustainability and the corporation." Journal of Management Inquiry 5, no. 2: p. 104119. 